\title{
Corneodesmosin: Structure, Function and Involvement in Pathophysiology
}

\author{
Nathalie Jonca, Cécile Caubet, Marina Guerrin, Michel Simon and Guy Serre* \\ CNRS - University Toulouse III, UMR 5165 "Epidermis Differentiation and Rheumatoid Autoimmunity", IFR 150 \\ (INSERM-CNRS-University Toulouse III-CHU), France
}

\begin{abstract}
Corneodesmosin (CDSN) was identified in the early $90^{\text {th }}$ by raising monoclonal antibodies against human plantar stratum corneum. It is a protein specific to desmosomes that will undergo transformation into corneodesmosomes, i.e. in man, desmosomes of the epidermis, of the three epithelial layers of the inner root sheath of the hair follicles and of the hard palate epithelium. After its secretion by granular keratinocytes via the lamellar bodies, CDSN is incorporated into the desmoglea of the desmosomes shortly before their transformation into corneodesmosomes. CDSN displays adhesive properties, mostly attributable to its N-terminal glycine-rich domain, and is sequentially proteolyzed as corneocytes migrate towards the skin surface. The recent inactivation of $C d s n$ in mice induced a lethal epidermal barrier disruption and hair follicle degeneration related to desmosome dysfunction, confirming the essential role of the protein in epidermis and hair follicle integrity. CDSN is located on chromosome 6, in the major psoriasis susceptibility locus PSORS1. Intriguingly, the only monogenic disease identified so far associated with nonsense mutations in $C D S N$, leading to the formation of a truncated protein, is a rare autosomal dominant disease, hypotrichosis simplex of the scalp. In this review, we expose data from the discovery of the protein to the most recent findings related to the relationship between its structure and function. In particular, the important benefits of mouse models and human diseases for the comprehension of CDSN role in the epidermis and hair follicles are reported in details.
\end{abstract}

Keywords: Corneodesmosome, corneodesmosin, cornification, desquamation, differentiation, epidermis, hyperkeratosis, hypotrichosis, kallikrein, psoriasis, stratum corneum.

\section{INTRODUCTION}

The aim of epidermal differentiation is to produce an efficient barrier separating the body from the environment. Such a role is fulfilled by the most superficial part of the epidermis, the stratum corneum (SC). This layer is composed of corneocytes, which are dead and flattened "mummified" cells endowed with a cornified envelope that provides extreme individual resistance. Owing to its relative impermeability to water and water soluble substances, the SC provides an efficient "inside-out" and "outside-in" barrier. Its physical resistance and strong cohesion, combined with its constant renewal, also offers a barrier against physical, chemical and microbial agents from the environment. In the $80^{\text {th }}$, the predominant model to explain the barrier function of the SC was the "brick wall" model proposed by P. Elias, in which the corneocytes were the bricks endowed within an intercellular substance composed mostly of lipids. In the early $90^{\text {th }}$, emerging data suggested that the lipids, crucial for the barrier's impermeability, played only a limited part in tissue cohesion, whereas the integrity of the SC depended mostly on the corneocytes and associated intercellular junctions. In particular, the finding that protease activity was necessary for desquamation to take place [1, 2], as well as the identification of corneodesmosin (CDSN) as the only protein specifically localized in the extracellular part of corneodesmosomes [3], the modified

*Address correspondence to this author at the CNRS-UPS UMR5165, CHU Purpan, Place du Docteur Baylac, TSA 40031, 31059 Toulouse Cedex 9, France; Tel: 3356115 8402; Fax: 3356149 9036;

E-mail: guy.serre@udear.cnrs.fr desmosomes in the SC, contributed to reconsider the place of proteins in the barrier function of the SC.

This review focuses on CDSN. We expose the data from the discovery of the protein to the more recent findings related to the relation between the structure and function of CDSN. In particular, the important benefits of mouse models and human diseases for the comprehension of the primordial role of CDSN in the epidermis and hair follicles are reported in details.

\section{CDSN: DISCOVERY OF A NEW PROTEIN SPECIFIC TO THE LATE STEPS OF EPIDERMAL DIFFER- ENTIATION}

In the early 90's, Guy Serre's group raised monoclonal antibodies against plantar SC in order to identify new late markers of epidermal differentiation. This led to the identification of CDSN, specifically recognized by two monoclonal antibodies, G36-19 and F28-27. Immunohistological studies indicated that CDSN is mainly expressed in cornified epithelia, and is absent from the non-cornified epithelia of the vagina, uterine cervix, or esophagus [3]. In addition, CDSN is present in the thymus Hassal's bodies, containing epithelial cells very similar to differentiated epidermal keratinocytes, and the $C D S N$ gene was shown to be transcribed in the placenta by RT-PCR experiments [4]. CDSN is also expressed in the hair follicle. The protein appears asynchronously in the 3 compartments of the inner root sheath, as their cells cornify: first in the Henle's layer, then in the cuticle, and lastly in the Huxley's layer $[3,5]$. CDSN is also clearly detected in cells of the hair follicle medulla [4]. 
In the epidermis, as shown by immunohistology, CDSN is cytoplasmic in the lower stratum granulosum ( $\mathrm{SG}$ ) then it becomes pericellular and progressively disappears in the lower SC. In contrast, the protein persists up to the desquamating keratinocytes in the palmoplantar epidermis and hard palate epithelium, with a discontinuous pericorneocyte microgranular distribution. Similarly, CDSN is detected as surface spots on corneocytes isolated by scraping the hard palate or the epidermis. Immunoelectron microscopy reveals that CDSN is present in the lamellar bodies from their emergence in the upper SG, in the extracellular space of the desmosomes of the granular keratinocytes and lastly in the core of the modified desmosomes of the SC. Finally, CDSN was shown to be largely preserved at the surface of purified cross-linked envelopes, where it is located on fibrils gathered over the external side (Fig. 1). Moreover, as the envelopes were purified under reducing and denaturing conditions, these data demonstrate that CDSN is covalently linked to the cornified envelope not only by disulfide bonds but also by other covalent links. The nature of these covalent links, the molecular partner -protein or lipid- involved in these links, as well as the enzyme responsible for the crosslink, remain unknown. Transglutaminases 1, 3 and 5 are responsible for the crosslink of the envelope precursor in the granular keratinocyte. However, these enzymes are located in the cytosol or anchored to the internal side of the cytoplasmic membrane. No transglutaminase isoform was described to be secreted in the extracellular space, where it could use CDSN as a substrate. CDSN is the first and until now the only protein specifically localized in the extracellular core of corneodesmosomes. This discovery contributed to the idea that corneodesmosomes are not only desmosome remnants, as considered until then, but fulfill a precise function in the upper layers of the epidermis.

CDSN is a $52-56 \mathrm{kDa}$ basic phosphoprotein. Deglycosylation experiments, reactivity to lectins, and chromatography on concanavalin A-sepharose indicated that it is N-glycosylated, with the oligosaccharide moiety comprising $\sim 10 \%$ of the protein mass [6]. Cloning of its
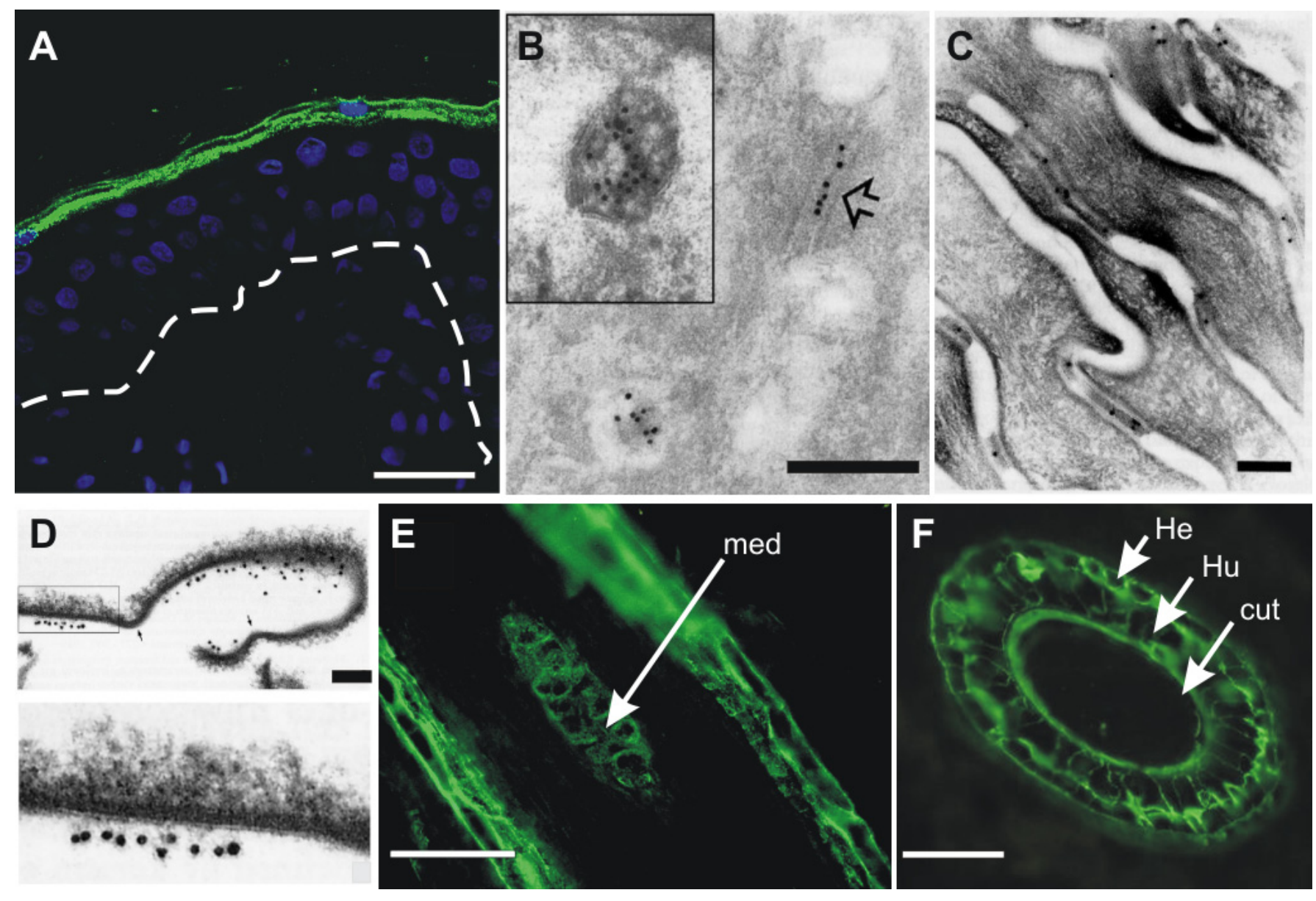

Fig. (1). Localization of CDSN in human epidermis and hair follicle. (A) Immunolocalization of CDSN in human breast epidermis by indirect immunofluorescence. (B-D). Immunoelectron microscopy analysis reveals CDSN in lamellar bodies and in the intercellular part of desmosomes (arrow) of the granular keratinocytes (B) as well as in the core of corneodesmosomes in the SC (C). CDSN is also bound to the cornified cell envelopes (D). (E, F). Immunofluorescence staining on transversal (E) and longitudinal (F) sections of hair follicles shows a restricted labeling of CDSN in the three compartments of the inner root sheath: Henle's layer (He), Huxley's layer (Hu) and cuticle (cut), and in the medulla (med). Scale bars: $50 \mu \mathrm{m}(\mathbf{A}), 200 \mathrm{~nm}(\mathbf{B}, \mathbf{C}), 100 \mathrm{~nm}(\mathbf{D}), 20 \mu \mathrm{m}(\mathbf{E}, \mathbf{F})$. In (A), nuclei were counterstained with Toto-3; dotted line, dermoepidermal junction. From references [3, 4] and unpublished data. Scale bars: $50 \mu \mathrm{m}(\mathbf{A}), 200 \mathrm{~nm}(\mathbf{B}, \mathbf{C}), 100 \mathrm{~nm}(\mathbf{D}), 20$ $\mu \mathrm{m}(\mathbf{E}, \mathbf{F})$. In (A), nuclei were counterstained with Toto-3; dotted line, dermoepidermal junction. From references [3, 4] and unpublished data. 
encoding cDNA revealed that CDSN is located on chromosome 6, in PSORS1, the major locus for psoriasis susceptibility. The mRNA encodes a 529-amino acid protein with a N-terminal signal peptide and one putative Nglycosylation site, consistent with the demonstration that it is secreted and glycosylated [7].

As a step in elucidating the mechanisms of tissue-specific expression, the human $C D S N$ promoter was characterized using transgenic mice. The transgene consisted on the $4.2 \mathrm{~kb}$ upstream region of the human $C D S N$ transcription initiation site linked to the $L a c Z$ gene. The reporter gene expression was detected by histoenzymology in special areas of the inner root sheath of the hair follicles and the hair medulla, with an expression pattern that perfectly matched that of the endogenous protein. The $4.2 \mathrm{~kb}$ DNA fragment is thus sufficient to correctly drive the expression of the reporter gene in the hair follicle. Surprisingly, no $\beta$-galactosidase activity was detected in the granular keratinocytes of the epidermis. However, induction of epidermal hyperproliferation either by pharmacological agents in topic or by wounding, led to strong expression of the reporter gene in the keratinocytes of the SG and in the parakeratotic corneocytes of the SC. This suggests that the transgene did not comprise all the elements necessary for the expression of CDSN in normal epidermis [4].

\section{CDSN IS AN ADHESIVE PROTEIN}

A striking feature of CDSN is its very high serine and glycine content $(27.5 \%$ and $16 \%$, respectively), particularly at both termini of the protein, i.e. amino acids $60-171$ and 375-450. Similar serine- and glycine-rich domains described at both ends of keratins have been proposed to form structural motifs called "glycine loops" as a consequence of the association of interspersed aromatic or aliphatic residues. These glycine loop domains have been suggested to mediate intermolecular adhesion by acting like a Velcro [8]. Such molecular interactions could be essential for skin homeostasis since mutations in the glycine loops of keratins and loricrin lead to various human cutaneous diseases, e.g., ichtyosis hystrix and palmoplantar keratodermas [9-12].

The potential role of CDSN in homophilic cell-cell interactions was assessed with mouse L-fibroblasts expressing a chimeric protein composed of the human CDSN and the transmembrane and cytoplasmic domains of mouse E-cadherin. CDSN thus anchored at the cell surface mediates cellular aggregation. Overlay binding assays and quantitative analysis by surface plasmon resonance using bacterial recombinant forms of full-length CDSN confirmed the homophilic adhesive properties of the protein [13, 14]. Moreover, recombinant CDSN associates into large homooligomers of at least three subunits that are only partially dissociated in $8 \mathrm{M}$ urea. These very stable oligomers obtained in vitro possibly correspond to cis- and/or transinteractions in vivo. Similar experiments of size-exclusion chromatography and surface plasmon resonance performed with truncated forms of recombinant CDSN showed that the N-terminal glycine loop domain, that perfectly matches features of the Steinert's glycine loop model, was necessary and sufficient, and thus responsible for both the oligomerization of CDSN and its homophilic adhesive properties. Altogether, these data clearly establish that
CDSN displays adhesive properties mediated by its $\mathrm{N}-$ terminal glycine loop domain.

\section{THE PROGRESSIVE PROTEOLYSIS OF CDSN IN THE STRATUM CORNEUM IS A PRE-REQUISITE FOR DESQUAMATION}

A tight balance between keratinocyte proliferation in the basal layer and cell shedding at the surface of the epidermis is essential for skin homeostasis and renewing of the SC protective barrier. Ultrastructural studies demonstrated that the degradation of corneodesmosomes in the outermost corneocytes is concomitant to desquamation, and showed that proteolytic cleavage of the extracellular part of these cell-cell adhesive structures is a key event in this process. In non-palmoplantar SC, normal exfoliation of corneocytes results from 2 steps of corneodesmosomal degradation. First, the non-peripheral corneodesmosomes are degraded at the interface between the stratum compactum and the stratum disjunctum. Second, the persistent peripheral corneodesmosomes are broken at the skin surface allowing corneocyte separation. Desquamation in palmoplantar skin is quite different, as the non-peripheral corneodesmosomes appear to be preserved against proteolysis in the lower SC accounting for the formation of a thick cornified layer, characteristic of these anatomic regions. This persistence is also a common feature of ichthyosis $[15,16]$.

The presence of cleavage products of CDSN in extracts of superficial SC attests to the degradation of corneodesmosomes. When extracted from viable layers of human epidermis, CDSN showed an apparent molecular mass of around $52-56 \mathrm{kDa}$, whereas a molecular form of 33 $\mathrm{kDa}$ is the major form extracted from the most superficial and less firmly attached corneocytes [3]. The presence of CDSN in the extracellular core of corneodesmosomes, and the in vitro demonstration of the homophilic adhesive properties of the protein, strongly suggest that, in vivo, CDSN contributes to the strong cohesion between corneocytes. Consequently, its proteolysis was proposed as one of the major biochemical changes that lead to desquamation [17].

Deglycosylation experiments and reactivity with lectins demonstrated that CDSN carbohydrate moiety does not prevent the protein proteolysis. Using a set of affinitypurified anti-peptide antibodies and monoclonal antibodies recognizing the five structural domains of CDSN, a refined characterization of its proteolysis during terminal differentiation of epidermis was realized. Immunoblotting, immunohistochimistry and immunoelectron microscopy experiments revealed that CDSN processing begins in the SG. In an initial proteolysis step, the $\mathrm{N}$ - and C-terminus of the protein are eliminated, giving rise to a $48-46 \mathrm{kDa}$ CDSN fragment that incorporates into desmosomes and might contribute to their transformation in corneodesmosomes. This fragment is still endowed with the glycine loop-related domains. Later, at the transition between the stratum compactum and the stratum disjunctum, cleavage of the $\mathrm{N}$ terminal adhesive glycine loop domain of the protein leads to a 36-30 kDa fragment. This step seems to be correlated with the abrupt decrease in SC cohesion. Finally, a CDSN fragment of only $15 \mathrm{kDa}$, mainly corresponding to the central part of the protein and devoid of both $\mathrm{N}-$ and $\mathrm{C}-$ 

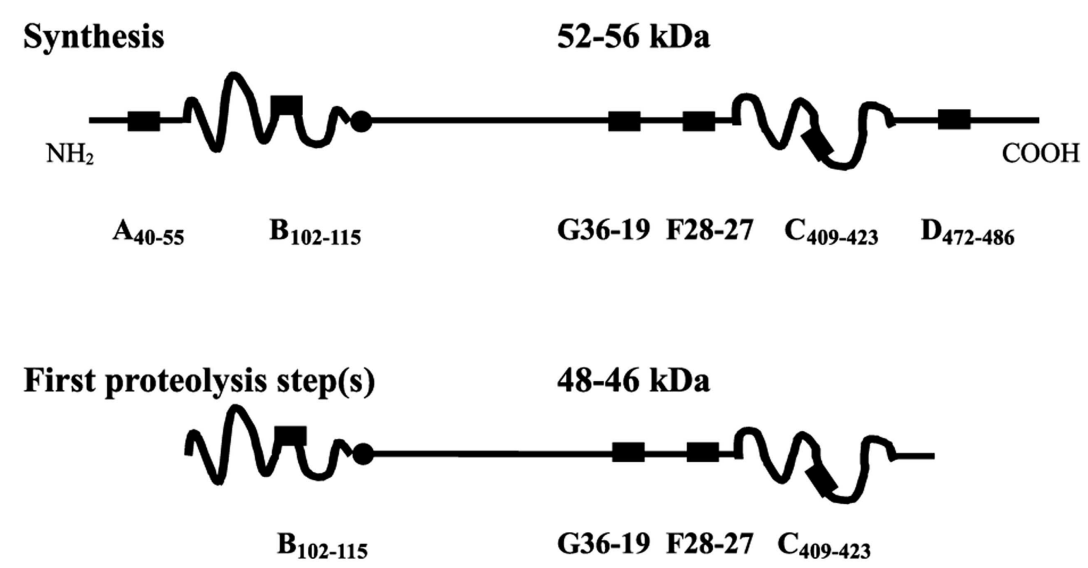

Second proteolysis step(s)

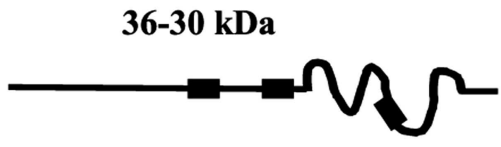

G36-19 F28-27 $\mathrm{C}_{409-423}$

Last proteolysis step(s)

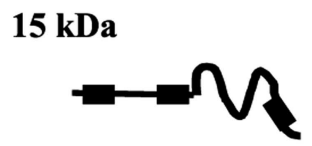

G36-19 F28-27 $\mathbf{C}_{409-423}$

Fig. (2). Schematic representation of CDSN proteolysis during the terminal differentiation of epidermis. A schema of CDSN proteolysis from the $52-56 \mathrm{kDa}$ protein to a fragment of $15 \mathrm{kDa}$ is proposed as deduced from immuno-ultrastructural and biochemical data. The apparent molecular mass of the various CDSN forms are indicated, the position of the epitopes recognized by the anti-CDSN antipeptide sera $\left(\mathrm{A}_{40-55}, \mathrm{~B}_{102-115}, \mathrm{C}_{409-423}\right.$, and $\left.\mathrm{D}_{472-486}\right)$ and monoclonal antibodies (G36-19 and F28-27) are shown by black boxes. The glycine loop-related domains and the N-glycosylation site of CDSN are represented by wavy lines and a black dot, respectively. From reference [18].

terminal glycine-rich domains, is present at the surface of the non-cohesive corneocytes [6, 18] (Fig. 2).

A number of different proteases of the serine, cysteine, or aspartic protease families, as well as protease inhibitors of these classes of enzymes, have been identified in the SC and might play a role in desquamation (for a review see [19-21]). Among these proteases, KLK7 and KLK5 - also known as stratum corneum chymotryptic enzyme (SCCE) and stratum corneum tryptic enzyme (SCTE), respectively- are serine proteases of the kallikrein family [22]. They are both highly expressed in granular keratinocytes and present in the intercellular spaces of the SC. Synthesized as inactive proforms, they are both activated by cleavage of a short Nterminal domain performed by an enzyme with the characteristics of a trypsin-like protease $[23,24]$. They were proposed to be actors of a cascade of activated proteases and inactive pro-enzymes that could regulate the rate of desquamation [25] (for a review see [21]). The importance of $\mathrm{pH}$ for $\mathrm{SC}$ cohesion and desquamation was also evidenced using superbases topically applied on hairless mouse skin [26]. CDSN was demonstrated to be a preferred substrate of both serine proteases in vitro [18]. A detailed analysis of the proteolysis of CDSN, but also of two other major components of the extracellular core of corneodesmosomes, namely desmocollin 1 (DSC1) and desmoglein 1 (DSG1), by KLK5 and KLK7 was realized. KLK7 directly cleaved CDSN and DSC1 but was unable to degrade DSG1. Incubation with KLK5 induced degradation of the three corneodesmosomal components. Moreover, it was shown that KLK7 and KLK5 conserved their activity at $\mathrm{pH}$ 5.6, close to that of physiological conditions in the SC. This study also suggested that KLK5 was able to activate the proform of KLK7 [27].

It is highly probable that, besides serine proteases, the involvement of other classes of proteases, also present in the SC, contribute to corneodesmosomal components degradation. Among these are the 2 cysteine proteases, Cathepsin L2 (CTSL2, also named Cathepsin V) and Cathepsin L-like (CTSL-like), and one aspartic acid protease Cathepsin D (CTSD). Synthesized as pro-enzymes, cathepsins undergo proteolytic maturation, sometimes in an autocatalytic way, and are active in an acidic environment. They have long been classified as hydrolases with exclusive functions in terminal degradation of proteins in the lysosomal compartment. Active forms of CTSL-like and CTSD have been shown to cleave CDSN in vitro $([28,29]$ and unpublished results). A detailed description of proteases and protease inhibitors, as well as recent data on the 
degradation of the corneodesmosomal components and on desquamation, leading to a model of regulation of corneodesmosomal components degradation, has been recently reviewed elsewhere [20].

\section{ESSENTIAL ROLE OF CDSN: LESSONS FROM ANIMAL MODELS}

The demonstration that CDSN is an adhesive protein covalently linked to the cornified envelope, as well as the data showing that its sequential proteolysis seems to be a prerequisite to desquamation, strongly suggest that CDSN is primordial for corneodesmosome function. But how exactly the protein fulfils its function remains unclear. CDSN was proposed to be necessary for corneodesmosome morphogenesis. It could also protect the other corneodesmosomal components from premature degradation by the proteases involved in desquamation. Finally, CDSN could reinforce cohesion by its own adhesive properties. Analysis of mouse models with an inactivated Cdsn gene is of particular interest to answer these questions. The first publication of $C d s n$ inactivation in mice, obtained by insertional mutation, concluded that Cdsn is necessary for corneodesmosome formation [30]. We also realized conditional ablation of Cdsn using the K14-driven Cremediated recombination. The detailed analysis of our $C d s n$ deficient mice confirmed that Cdsn is vital for epidermal barrier and hair follicle integrity, and our conclusion supported the idea that Cdsn is mainly an adhesive protein [31]. Immediately after birth, $\mathrm{KO}$ neonates showed a severe skin detachment starting from ventral area and extremities (paws and snoot), and died within $1 \mathrm{~h}$. In contrast, heterozygous newborns did not develop any skin phenotype, demonstrating that ablation of only one allele had no consequences. Surprisingly, KO pups obtained after caesarian delivery at E18.5 were indistinguishable from their littermates. However, during grooming by surrogate mother, they rapidly developed a skin phenotype similar to that observed after natural birth (Fig. 3). The effects of the mutation on skin permeability were analyzed by dye penetration assay and transepidermal water loss measurements, and the mechanical resistance of the skin was evaluated by quantification of SC proteins obtained by tape stripping. The results showed that, although the greatly reduced mechanical resistance of the $\mathrm{SC}$ is an intrinsic feature caused by $C d s n$ deficiency, epidermal tear, leading to lethal barrier defect, occurred only under mechanical stress encountered after birth.

Intact dorsal skin of $C d s n \mathrm{KO}$ newborns (i.e. before appearance of the macroscopic phenotype) observed by transmission electron microscopy revealed a similar organization and thickness of the granular layer and the SC than WT skin. In particular, we did not detect any significant differences in the number of transitional desmosomes between WT and KO neonates, unlike Matsumoto and coworkers' published data [30]. Moreover, the electron density of the corneodesmosomes appeared unchanged, suggesting no premature degradation of these structures. Thus, Cdsn does not seem indispensable for the morphogenesis of corneodesmosomes nor their protection from SC proteases. On the other hand, the histological analysis of dorsal $\mathrm{KO}$ skin revealed the presence of blisters as soon as cornification occurs, that is, at the SG/SC transition. At the ultrastructural level, the main part of numerous split junctions typically remain attached to the granular keratinocytes, suggesting that the cohesive defect lies in the upper side of the junction (Fig. 4). Thus, although Cdsn was already present in the extracellular core of desmosomes from the SG, it seemed to play its fundamental role only when cornification is complete. Intriguingly, no thinning out of single or bundled corneocytes throughout the $\mathrm{SC}$ was observed but detachment of the whole SC from the subjacent SG. This suggested that the SG/SC interface may actually be the most fragile zone as it links two epidermal layers with different junctional organizations and mechanical characteristics: desmosomes and keratin intermediate filaments organized in taut cables in the SG, rigid cornified envelopes linked by corneodesmosomes in the SC.
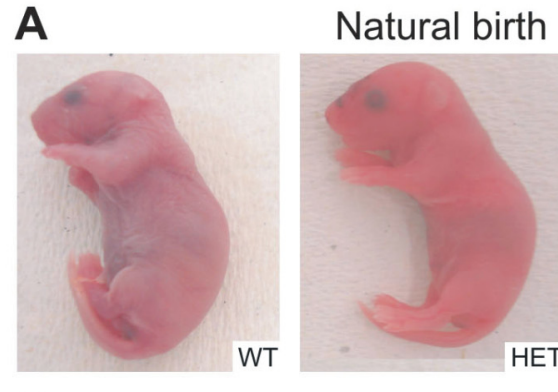

B
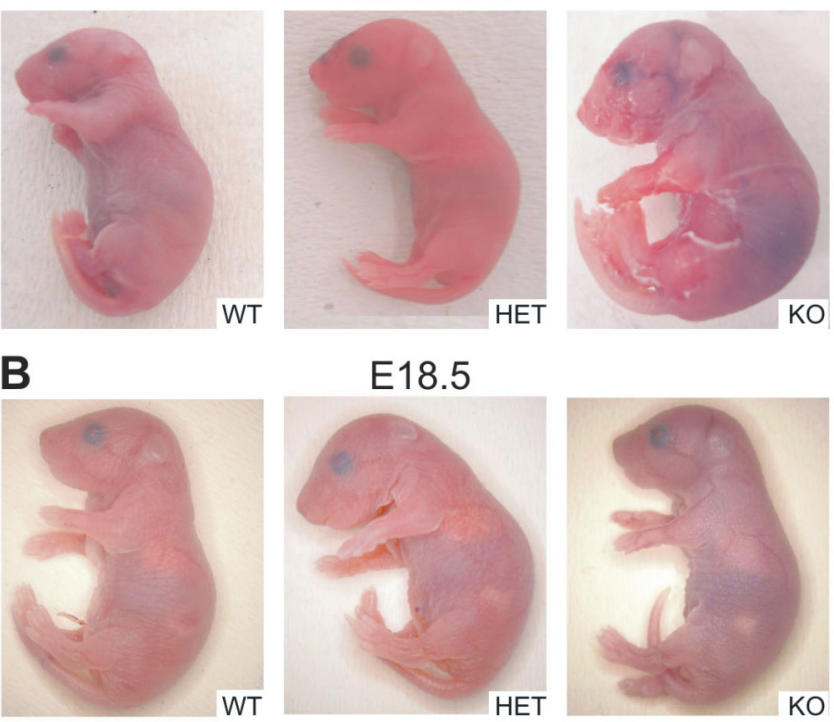

E18.5
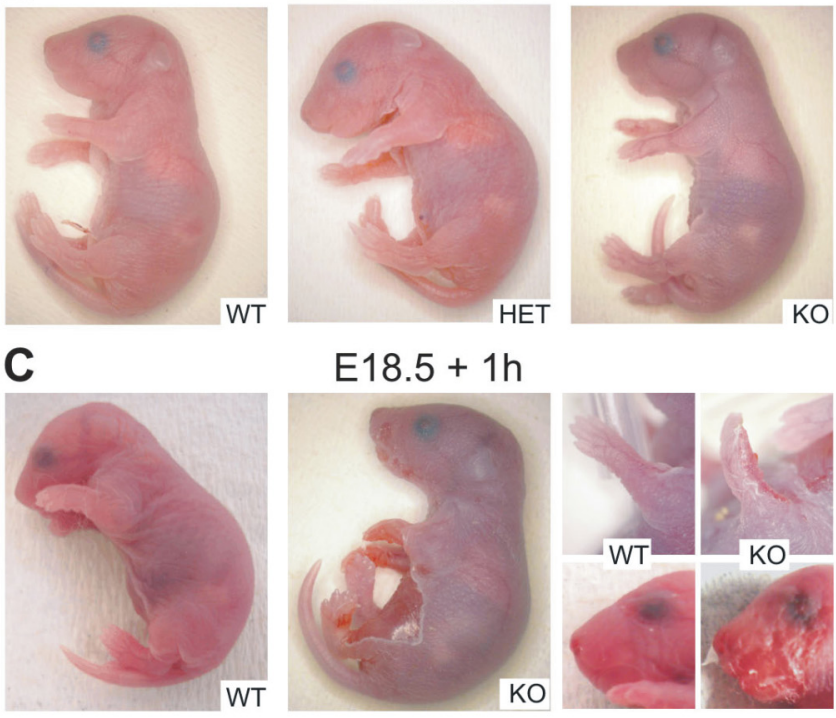

Fig. (3). Mice lacking Cdsn show a drastic skin tear leading to postnatal death. (A) Macroscopic appearance of wild type (WT), heterozygous (HET) and knockout (KO) pups born at full term. KO pups died within $1 \mathrm{~h}$ after birth and showed considerable skin detachment, particularly in the ventral area, paws and snout while HET newborns had no phenotype. (B) Macroscopic appearance of WT, HET and KO mice delivered by caesarian section at E18.5. Immediately after birth, $\mathrm{KO}$ neonates were indistinguishable from the WT and HET littermates. (C) When neonates were left in surrogate mother's care for $1 \mathrm{~h}$, the skin phenotype of KO pups became manifest and death occurred rapidly. Higher magnification of paw and snout of WT and KO neonates are shown on the right panel. From reference [31].

In order to assess long-term consequences of Cdsn inactivation, we performed grafting of skin from Cdsndeficient newborns onto nude mice. The grafted epidermis 

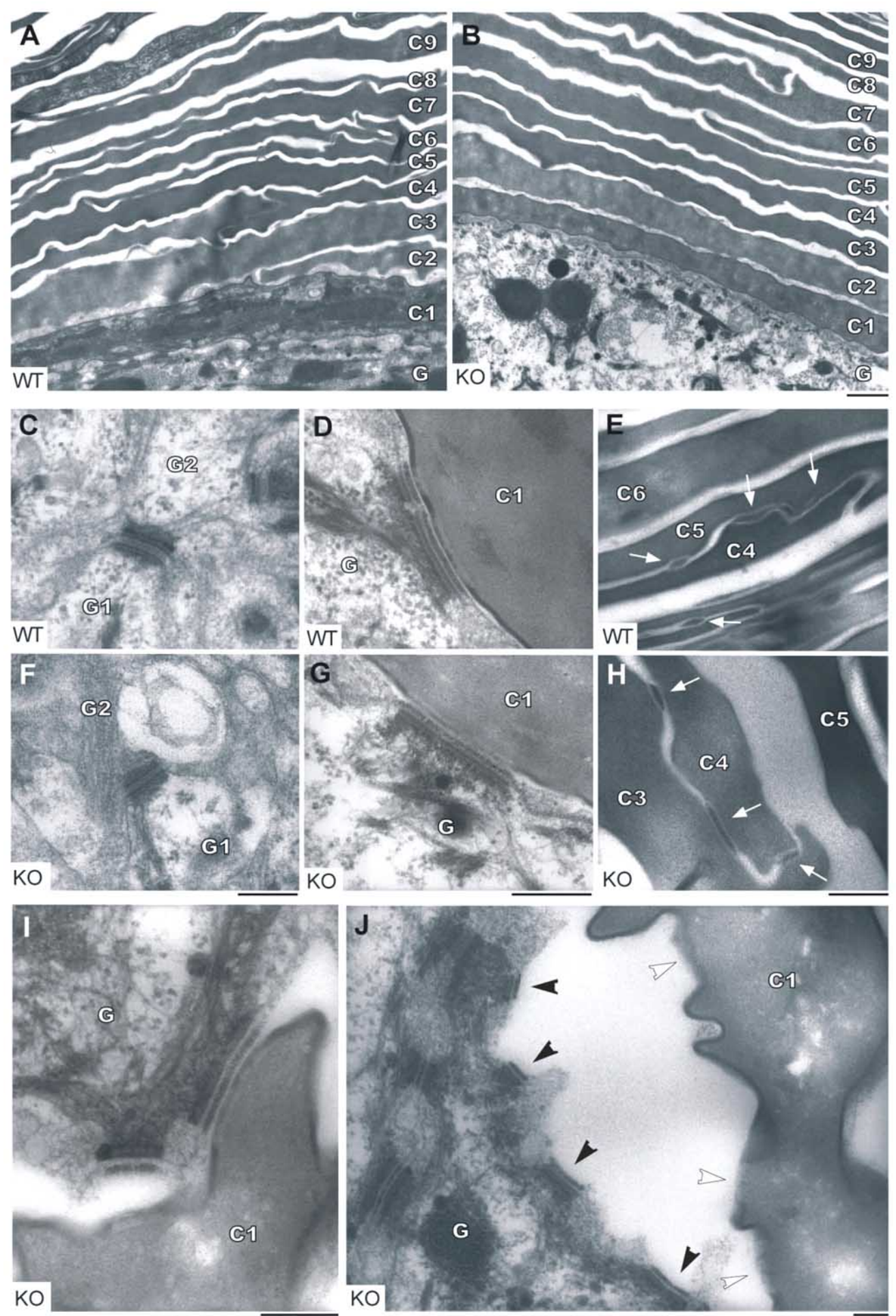

Fig. (4). Ultrastructural abnormalities in CDSN knockout neonate epidermis. Dorsal skin of WT and KO mice was processed for transmission EM. (A, B) At low magnification, the overall morphology of the cells at the junction between the granular and cornified layers shows no obvious abnormality. (G, G1, G2), granular keratinocytes; (C1) to (C9), corneocytes, with C1 being adjacent to the granular layer and C9 being nearest the skin surface. (C-H) The ultrastructure of desmosomes and corneodesmosomes from WT (C-E) and KO (F-H) epidermis was analyzed at higher magnification. Regular desmosomes in the SG (C, F), transitional desmosomes at the SG/SC interface (D, G), and corneodesmosomes (white arrows) in the SC (E, H), show the same classical morphology in WT and KO epidermis. (I, J) Detachment between granular keratinocyte and corneocyte is frequently observed in KO epidermis. Split desmosomes isolated (I) or adjacent (J) can be seen. The mid-dense intercellular plugs of the detached transitional desmosomes remain associated with the granular keratinocyte (black arrowhead) whereas an interruption in the electron dense line corresponding to the cornified envelope is clearly visible at the surface of the corneocyte, at the site where the desmosome was attached (white arrowhead). Bars: A, B $=2 \mu \mathrm{m}, \mathrm{C}-\mathrm{J}=0.2 \mu \mathrm{m}$. From reference [31]. 
first developed acanthosis and hyperkeratosis. Increased expression of various differentiation markers (involucrin, $\mathrm{K} 10$ ), and induced expression of the hyperproliferative keratin K6, confirmed the altered differentiation and the hyperproliferative state of the grafted epidermis. However, these compensatory mechanisms appeared to be ineffective, and were followed by a complete disappearance of the epidermis. Cdsn was thus necessary for maintaining the integrity and barrier function of the postnatal epidermis. The consequences of Cdsn loss in adult mice was investigated using an additional model consisting on K14-driven Cremediated loxP recombination system with a chimeric CreERT2 recombinase that can be induced by tamoxifen. Inducible $C d s n \mathrm{KO}$ resulted in histological abnormalities similar to that of the Cdsn KO skin graft model. Barrier restoration remained unsuccessful, compromising the vital prognosis of the mice when the whole skin area was affected. Thus, Cdsn seemed to play a vital role in adult epidermis too.

These somatic and inducible Cdsn KO mice also allowed to investigate the consequences of Cdsn loss on hair follicles. Analysis of skin sections from newborn Cdsn KO mice showed a similar morphology and number of hair follicles to those of skin from WT littermate, suggesting that Cdsn seemed to be dispensable for hair follicle morphogenesis. Long term consequences of Cdsn excision in hair follicle was analyzed after grafing of dorsal $C d s n \mathrm{KO}$ skin onto nude mice and in adult mouse skin induced for $C d s n$ excision. In both cases, hair follicles first showed an altered morphology, developed cysts, and finally disappeared. This suggested that Cdsn is necessary for maintaining normal hair follicle architecture.

Altogether, these data obtained in vivo demonstrated that Cdsn plays a vital role in the epidermal barrier and hair follicle integrity by preventing the rupture of corneodesmosome.

\section{CDSN AND HUMAN DISEASES}

A common feature of numerous skin disorders is scaling, usually associated with thickening of the SC. The hyperkeratosis may be due to increased cell proliferation, impaired desquamation, or a combination of both. Hyperkeratosis has been invariably associated with the persistence of both peripheral and non-peripheral corneodesmosomes in the outer SC, a feature reminiscent of normal palmoplantar epidermis. This has been accurately described in the case of several congenital ichthyoses [32, 33], winter xerosis [16] or soap-induced xerosis [19]. In parallel, increased amounts of CDSN were detected in all the analyzed hyperkeratotic lesions, including inflammatory diseases [15, 34]. Mutations in several different genes were identified as responsible for ichtyosis, but none of them, to date, corresponded to mutations in $C D S N$. As a defect in the barrier is commonly encountered in hyperkeratosis, CDSN over-expression occurring in the epidermis from affected individuals may rather result from a compensatory mechanism to the barrier defect.

CDSN is of particular interest in relation to psoriasis. Indeed, as previously mentioned, $C D S N$ is localized to chromosome 6p21 at PSORS1, the major susceptibility locus of this chronic inflammatory skin disorder. $C D S N$ is highly polymorphic, with more than one SNP every $100 \mathrm{bp}$ in the coding region [35]. Some of these SNPs have been associated with psoriasis in many genetic studies (reviewed in [36]). However, PSORS1 spans a $\sim 300 \mathrm{~kb}$ region containing at least 11 other genes including $H L A-C$, and the exact identity of the PSORS1 gene remains controversial owing to extensive linkage disequilibrium across this region. A recent study revealed that only 2 genes from the locus, $H L A-C$ and $C D S N$, yield alleles encoding protein unique to risk haplotypes [37]. However, this study and others, using ancestral haplotype analysis, resulted in contradictory findings for the PSORS1 gene: $H L A-C, C D S N$, or both genes [37-41]. Ultrastructural analyses of psoriatic skin show that desmosomes are not dramatically transformed into corneodesmosomes at the SG / SC transition, but rather stay at an intermediate state between the two structures. As already stated, persistence of corneodesmosomes accompanied by an increased amount of CDSN in the SC, is a feature common to hyperkeratotic diseases. However, the earlier synthesis and secretion of CDSN in the upper stratum spinosum, is specific to psoriasis among several hyperproliferative disorders [34]. Finally, persistence of fulllength CDSN and of other corneodesmosomal proteins in the upper SC was observed in psoriatic lesions [42]. The psoriasis-associated SNPs of CDSN could affect the function of the corresponding proteins. To date, only one study has shown that an intragenic SNP (SNP*971T), present in 3 common haplotypes associated with psoriasis in various ethnic groups, is responsible for an increased stability of the encoded mRNAs [43]. Although these results are in agreement with the known overexpression of CDSN in psoriatic lesions $[15,34]$, they pointed a discrepancy between CDSN haplotypes at the mRNA level only. The CDSN*TTC allele (SNP*619T, SNP*1236T, SNP*1243C), associated with the disease in many different ethnic groups, was identified as the smallest combination of protein altering variations unique to $C D S N$ carried by risk haplotypes [37]. Assessment of the functional consequence of these aminoacid substitutions should provide insight into CDSN putative involvement in psoriasis (Jonca et al., submitted).

Intriguingly, the only monogenic disease associated with mutations in $C D S N$ that has been identified so far revealed a hair phenotype and did not affect the epidermis. Indeed, nonsense mutations in $C D S N$ were found to be responsible for a rare autosomal dominant disease, hypotrichosis simplex of the scalp (HSS; OMIM 146520) [44]. Affected individuals experience progressive loss of scalp hairs beginning in the middle of the first decade and almost complete baldness by the third decade. The body hairs, beard, eyebrows and axillary hairs are normal. To date, 3 different nonsense mutations have been reported in 4 families from Israel, Denmark and Mexico [45]. All of them produce a truncated form of CDSN, mainly corresponding to the N-terminal glycine loop domain. An antibody directed against this domain labeled irregularly sized clusters located in ridges of the superficial dermis and at the periphery of the hair follicles deeper in the dermis. These aggregates did not stained with several antibodies directed against epithelial proteins (like keratins K1, K2, K1O, K11, (pro)filaggrin or involucrin) strongly implying that in these aggregates the truncated CDSN is not associated with any of these proteins. By western blot analysis of dermis extracts, the truncated 
CDSN was detected as SDS- and boiling-resistant dimers, trimers and larger oligomers [44].

We noticed histological similarities between HSS and the two main forms of primary localized cutaneous amyloidosis, namely lichen amyloidosis and macular amyloidosis, two rare chronic pruritic skin disorders. Amyloidoses form a large heterogeneous group of misfolding diseases associated with the conversion of soluble peptides/proteins into highly organized fibrillar aggregates [46]. Shape, tinctorial properties and secondary structure of amyloid fibrils are characteristic: the fibrils are unbranched, 7.5-20-nm thick, bind Congo Red and Thioflavine $\mathrm{T}$ dyes, exhibit yellowgreen birefringence in polarized light upon binding of Congo Red, and have a significant content of $\beta$-sheets that are systematically hydrogen-bonded. In both lichen amyloidosis and macular amyloidosis, amyloid material that could be derived from epidermal keratins accumulates in the dermal papillae [46-52]. With regard to the histological similarities between HSS and these two localized cutaneous amyloidoses, we wondered if HSS was indeed an amyloidosis (Caubet et al., submitted). Consistent with the possibility that the mutant CDSN may adopt an amyloid conformation, the dermal deposits from skin biopsies of affected individuals were stained by Thioflavine $\mathrm{T}$ and Congo Red. The presence of the SAP component, a protein that colocalizes with amyloid plaques, irrespective of their chemical nature and the clinical type of amyloidosis, was also evidenced within CDSN deposits. In agreement with these in vivo observations, analysis by electron microscopy demonstrated that recombinant forms of the truncated mutated CDSN expressed in the skin of HSS patients, as well as the recombinant N-terminal glycine/serine rich domain of CDSN alone, did assemble into ring-shaped oligomeric structures and into fibrils. The amyloid-like structure of the fibrils was evidenced by tinctorial affinity for Congo Red and Thioflavine $\mathrm{T}$ dyes. The recorded Fourier transform infrared spectra suggested that the recombinant $\mathrm{N}$ terminal glycine/serine rich domain of CDSN assembly into fibrils was accompanied by a conformational change with a very significant increase in $\beta$-sheet content typical of the assembly of native unfolded polypeptides into amyloid fibrils. Finally, the truncated CDSN forms expressed in the skin of HSS patients, and assembled into pre-fibrillar oligomers, were toxic to primary keratinocytes grown in vitro. This was consistent with two recent reports suggesting that soluble oligomeric species rather than mature amyloid fibrils were the toxic entity in amyloid-related diseases [53, 54].

Mouse models with inactivation of $C d s n$ showed that heterozygous $\mathrm{Cdsn}^{+/-}$mice were indistinguishable from their wild type littermate. In particular, they did not develop any hair phenotype up to 8 months. This reinforced the hypothesis that HSS is not caused by Cdsn haploinsufficiency $[30,31]$. Given our previous finding that a recombinant truncated form of CDSN is able to bind the entire CDSN [13, 14], a dominant negative interaction between the mutant and wild-type proteins may account for the loss of cohesion in the inner root sheath of the hair follicles. This could affect the functionality of the inner root sheath, such as a decrease in diffusion barrier function resulting in anagen to catagen transition and perturbation of a hair cycle reinitiation. However, in view of the delayed onset of alopecia and the fact that lost hairs are not regenerated, another possibility is that the observed CDSN aggregates are toxic for the hair follicle cells. The aggregates released from defective hair follicles probably migrate through the dermis until they reach the dermo-epidermal junction under which they accumulate. As similar aggregates are not detected in the epidermis, these are probably degraded by SC proteases.

The results from our observations on affected individual skin biopsies, together with the data from experiments performed in vitro using recombinant mutant forms of CDSN or the N-terminal glycine/serine rich domain of CDSN alone revisits HSS as a possible amyloidosis, among the growing list of these human diseases.

\section{CONCLUSION - FUTURE PROSPECTS}

The data accumulated from the discovery of CDSN to the more recent works using mouse models or studying human diseases led to the comprehension of the primordial role of CDSN in epidermis and hair follicle integrity. However, some functional aspects concerning CDSN are not fully understood, especially at the molecular level. For instance, immunoelectron microscopy experiments clearly demonstrate that CDSN exclusively localizes to the extracellular core of corneodesmosomes in the SC. But how is this specific localization achieved? It is likely that a preexisting component of the extracellular part of the junction interacts with CDSN. We were unable to evidence any interaction between CDSN and desmosomal cadherins (unpublished results). The molecular partner for CDSN thus remains to be identified. Similarly, the molecular event, probably involving transglutaminase-like enzymatic activity, and resulting in the covalent link of CDSN to the cornified envelope, has not yet been characterized. Finally, the underlying molecular mechanism by which CDSN assumes its adhesive function within the corneodesmosome has not been fully elucidated. The previously demonstrated homophilic adhesive properties of CDSN and the strong resistance of aggregates formed by bacterially recombinant CDSN to highly denaturing conditions $[13,14]$ suggested that, in vivo, CDSN reinforced cohesion by its own adhesive properties. However, adhesion provided by glycine-loop domains has been suggested to mediate reversible and constantly adjustable intermolecular links [8]. Consistent with this, CDSN could give the junction the elasticity essential to prevent breaking as soon as the cell envelope rigidifies. Clarification of those molecular aspects of CDSN is the task for the future.

\section{REFERENCES}

[1] Bissett DL, McBride JF, Patrick LF. Role of protein and calcium in stratum corneum cell cohesion. Arch Dermatol Res 1987; 279: 1849.

[2] Lundstrom A, Egelrud T. Stratum corneum chymotryptic enzyme: a proteinase which may be generally present in the stratum corneum and with a possible involvement in desquamation. Acta Derm Venereol 1991; 71: 471-4.

[3] Serre G, Mils V, Haftek M, et al. Identification of late differentiation antigens of human cornified epithelia, expressed in re-organized desmosomes and bound to cross-linked envelope. J Invest Dermatol 1991; 97: 1061-72.

[4] Gallinaro H, Jonca N, Langbein L, et al. A $4.2 \mathrm{~kb}$ upstream region of the human corneodesmosin gene directs site-specific expression in hair follicles and hyperkeratotic epidermis of transgenic mice. $\mathrm{J}$ Invest Dermatol 2004; 122: 730-8. 
[5] Mils V, Vincent C, Croute F, Serre G. The expression of desmosomal and corneodesmosomal antigens shows specific variations during the terminal differentiation of epidermis and hair follicle epithelia. J Histochem Cytochem 1992; 40: 1329-37.

[6] Simon M, Montezin M, Guerrin M, Durieux JJ, Serre G. Characterization and purification of human corneodesmosin, an epidermal basic glycoprotein associated with corneocyte-specific modified desmosomes. J Biol Chem 1997; 272: 31770-6.

[7] Guerrin M, Simon M, Montezin M, Haftek M, Vincent C, Serre G. Expression cloning of human corneodesmosin proves its identity with the product of the $\mathrm{S}$ gene and allows improved characterization of its processing during keratinocyte differentiation. J Biol Chem 1998; 273: 22640-7.

[8] Steinert PM, Mack JW, Korge BP, Gan SQ, Haynes SR, Steven AC. Glycine loops in proteins: their occurrence in certain intermediate filament chains, loricrins and single-stranded RNA binding proteins. Int J Biol Macromol 1991; 13: 130-9.

[9] Maestrini E, Monaco AP, McGrath JA, et al. A molecular defect in loricrin, the major component of the cornified cell envelope, underlies Vohwinkel's syndrome. Nat Genet 1996; 13: 70-7.

[10] Sprecher E, Ishida-Yamamoto A, Becker OM, et al. Evidence for novel functions of the keratin tail emerging from a mutation causing ichthyosis hystrix. J Invest Dermatol 2001; 116: 511-9.

[11] Terrinoni A, Puddu P, Didona B, et al. A mutation in the V1 domain of K16 is responsible for unilateral palmoplantar verrucous nevus. J Invest Dermatol 2000; 114: 1136-40.

[12] Whittock NV, Smith FJ, Wan H, et al. Frameshift mutation in the V2 domain of human keratin 1 results in striate palmoplantar keratoderma. J Invest Dermatol 2002; 118: 838-44.

[13] Caubet C, Jonca N, Lopez F, Esteve JP, Simon M, Serre G. Homooligomerization of human corneodesmosin is mediated by its $\mathrm{N}$ terminal glycine loop domain. J Invest Dermatol 2004; 122: 747 54.

[14] Jonca N, Guerrin M, Hadjiolova K, et al. Corneodesmosin, a component of epidermal corneocyte desmosomes, displays homophilic adhesive properties. J Biol Chem 2002; 277: 5024-9.

[15] Haftek M, Simon M, Kanitakis J, et al. Expression of corneodesmosin in the granular layer and stratum corneum of normal and diseased epidermis. Br J Dermatol 1997; 137: 864-73.

[16] Simon M, Bernard D, Minondo AM, et al. Persistence of both peripheral and non-peripheral corneodesmosomes in the upper stratum corneum of winter xerosis skin versus only peripheral in normal skin. J Invest Dermatol 2001; 116: 23-30.

[17] Lundström A, Serre G, Haftek M, Egelrud T. Evidence for a role of corneodesmosin, a protein which may serve to modify desmosomes during cornification, in stratum corneum cell cohesion and desquamation. Arch Dermatol Res 1994; 286: 369-75.

[18] Simon M, Jonca N, Guerrin M, et al. Refined characterization of corneodesmosin proteolysis during terminal differentiation of human epidermis and its relationship to desquamation. J Biol Chem 2001; 276: 20292-9.

[19] Rawlings A. Trends in stratum corneum research and the management of dry skin conditions. Int J Cosmet Sci 2003; 25: 6395.

[20] Jonca N, Caubet C, Guerrin M, Simon M, Serre G. Corneodesmosomal proteins. In: Rawlings AV, Leyden JJ, Eds. Skin moisturization, $2^{\text {nd }}$ ed. New-York: Informa Healthcare USA, Inc; 2009; pp. 99-122.

[21] Ovaere P, Lippens S, Vandenabeele P, Declercq W. The emerging roles of serine protease cascades in the epidermis. Trends Biochem Sci 2009 (in press).

[22] Diamandis EP, Yousef GM, Luo LY, Magklara A, Obiezu CV. The new human kallikrein gene family: implications in carcinogenesis. Trends Endocrinol Metab 2000; 11: 54-60.

[23] Brattsand M, Egelrud T. Purification, molecular cloning, and expression of a human stratum corneum trypsin-like serine protease with possible function in desquamation. J Biol Chem 1999; 274 : 30033-40.

[24] Hansson L, Stromqvist M, Backman A, Wallbrandt P, Carlstein A, Egelrud T. Cloning, expression, and characterization of stratum corneum chymotryptic enzyme. A skin-specific human serine proteinase. J Biol Chem 1994; 269: 19420-6.

[25] Ekholm IE, Brattsand M, Egelrud T. Stratum corneum tryptic enzyme in normal epidermis: a missing link in the desquamation process? J Invest Dermatol 2000; 114: 56-63.
[26] Hachem JP, Crumrine D, Fluhr J, Brown BE, Feingold KR, Elias PM. $\mathrm{pH}$ directly regulates epidermal permeability barrier homeostasis, and stratum corneum integrity/cohesion. J Invest Dermatol 2003; 121: 345-53.

[27] Caubet C, Jonca N, Brattsand $\mathrm{M}$, et al. Degradation of corneodesmosome proteins by two serine proteases of the kallikrein family, SCTE/KLK5/hK5 and SCCE/KLK7/hK7. J Invest Dermatol 2004; 122: 1235-44

[28] Bernard D, Mehul B, Thomas-Collignon A, et al. Analysis of proteins with caseinolytic activity in a human stratum corneum extract revealed a yet unidentified cysteine protease and identified the so-called "stratum corneum thiol protease" as cathepsin 12 . J Invest Dermatol 2003; 120: 592-600.

[29] Igarashi S, Takizawa T, Takizawa T, et al. Cathepsin D, but not cathepsin E, degrades desmosomes during epidermal desquamation. Br J Dermatol 2004; 151: 355-61.

[30] Matsumoto M, Zhou Y, Matsuo S, et al. Targeted deletion of the murine corneodesmosin gene delineates its essential role in skin and hair physiology. Proc Natl Acad Sci U S A 2008; 105: 6720-4.

[31] Leclerc EA, Huchenq A, Mattiuzzo NR, et al. Corneodesmosin gene ablation induces lethal skin-barrier disruption and hair-follicle degeneration related to desmosome dysfunction. J Cell Sci 2009; 122: 2699-709.

[32] Elsayed-Ali H, Barton S, Marks R. Stereological studies of desmosomes in ichthyosis vulgaris. Br J Dermatol 1992; 126: 24-8.

[33] Ghadially R, Williams ML, Hou SY, Elias PM. Membrane structural abnormalities in the stratum corneum of the autosomal recessive ichthyoses. J Invest Dermatol 1992; 99: 755-63.

[34] Allen M, Ishida-Yamamoto A, McGrath J, et al. Corneodesmosin expression in psoriasis vulgaris differs from normal skin and other inflammatory skin disorders. Lab Invest 2001; 81: 969-76.

[35] Guerrin M, Vincent C, Simon M, Ahnini TR, Fort M, Serre G. Identification of six novel polymorphisms in the human corneodesmosin gene. Tissue Antigens 2001; 57: 32-8.

[36] Capon F, Novelli G, Semprini S, et al. Searching for psoriasis susceptibility genes in Italy: genome scan and evidence for a new locus on chromosome 1. J Invest Dermatol 1999; 112: 32-5.

[37] Nair RP, Stuart PE, Nistor I, et al. Sequence and haplotype analysis supports HLA-C as the psoriasis susceptibility 1 gene. Am J Hum Genet 2006; 78: 827-51.

[38] Capon F, Toal IK, Evans JC, et al. Haplotype analysis of distantly related populations implicates corneodesmosin in psoriasis susceptibility. J Med Genet 2003; 40: 447-52.

[39] Helms C, Saccone NL, Cao L, et al. Localization of PSORS1 to a haplotype block harboring HLA-C and distinct from corneodesmosin and HCR. Hum Genet 2005; 118: 466-76.

[40] Orrù S, Giuressi E, Carcassi C, Casula M, Contu L. Mapping of the major psoriasis-susceptibility locus (PSORS1) in a 70-Kb interval around the corneodesmosin gene (CDSN). Am J Hum Genet 2005; 76: 164-71.

[41] Veal CD, Capon F, Allen MH, et al. Family-based analysis using a dense single-nucleotide polymorphism-based map defines genetic variation at PSORS1, the major psoriasis-susceptibility locus. Am J Hum Genet 2002; 71: 554-64.

[42] Simon M, Tazi-Ahnini R, Jonca N, Caubet C, Cork MJ, Serre G. Alterations in the desquamation-related proteolytic cleavage of corneodesmosin and other corneodesmosomal proteins in psoriatic lesional epidermis. Br J Dermatol 2008; 159: 77-85.

[43] Capon F, Allen MH, Ameen M, et al. A synonymous SNP of the corneodesmosin gene leads to increased mRNA stability and demonstrates association with psoriasis across diverse ethnic groups. Hum Mol Genet 2004; 13: 2361-8.

[44] Levy-Nissenbaum E, Betz RC, Frydman M, et al. Hypotrichosis simplex of the scalp is associated with nonsense mutations in CDSN encoding corneodesmosin. Nat Genet 2003; 34: 151-3.

[45] Lai-Cheong JE, Arita K, McGrath JA. Genetic diseases of junctions. J Invest Dermatol 2007; 127: 2713-25.

[46] Stefani M. Protein misfolding and aggregation: new examples in medicine and biology of the dark side of the protein world. Biochim Biophys Acta 2004; 1739: 5-25.

[47] Apaydin R, Gurbuz Y, Bayramgurler D, Muezzinoglu B, Bilen N. Cytokeratin expression in lichen amyloidosus and macular amyloidosis. J Eur Acad Dermatol Venereol 2004; 18: 305-9.

[48] Chang YT, Liu HN, Wang WJ, Lee DD, Tsai SF. A study of cytokeratin profiles in localized cutaneous amyloids. Arch Dermatol Res 2004; 296: 83-8. 
[49] Kobayashi H, Hashimoto K. Amyloidogenesis in organ-limited cutaneous amyloidosis: an antigenic identity between epidermal keratin and skin amyloid. J Invest Dermatol 1983; 80: 66-72.

[50] Kumakiri M, Hashimoto K. Histogenesis of primary localized cutaneous amyloidosis: sequential change of epidermal keratinocytes to amyloid via filamentous degeneration. J Invest Dermatol 1979; 73: 150-62.

[51] Maeda H, Ohta S, Saito Y, Nameki H, Ishikawa H. Epidermal origin of the amyloid in localized cutaneous amyloidosis. $\mathrm{Br} \mathrm{J}$ Dermatol 1982; 106: 345-51.
[52] Masu S, Hosokawa M, Seiji M. Amyloid in localized cutaneous amyloidosis: immunofluorescence studies with anti-keratin antiserum especially concerning the difference between systemic and localized cutaneous amyloidosis. Acta Derm Venereol 1981; 61: 381-4.

[53] Baglioni S, Casamenti F, Bucciantini M, et al. Prefibrillar amyloid aggregates could be generic toxins in higher organisms. J Neurosci 2006; 26 : 8160-7.

[54] Bucciantini M, Calloni G, Chiti F, et al. Prefibrillar amyloid protein aggregates share common features of cytotoxicity. J Biol Chem 2004; 279: 31374-82.

(C) Jonca et al.; Licensee Bentham Open.

This is an open access article licensed under the terms of the Creative Commons Attribution Non-Commercial License (http://creativecommons.org/licenses/by-nc/ 3.0/) which permits unrestricted, non-commercial use, distribution and reproduction in any medium, provided the work is properly cited. 